\title{
Applying Digital Image Processing Technology in Discovering Green Patches in the Desert of Saudi Arabia
}

\author{
Ali Mehdi ${ }^{1}$, Md Alamin Bhuiyan ${ }^{2}$ \\ Department of Computer Engineering, College of Computer Sciences and Information Technology \\ King Faisal Universitym, P.O. Box 380, Al-Ahsa 31982, Saudi Arabia
}

\begin{abstract}
In recent years, the Kingdom of Saudi Arabia has witnessed a noticeable growth of grass and small trees in the desert, forming green patches. Those green patches may have the potential to spread and cover a wider area in the desert in the coming years, thus, making areas of the desert potential agricultural land. This research aims to detect the change of green patches in the desert of Saudi Arabia to solve the challenge that is mainly due to the lack of an organized dataset. Using a series of satellite images of the desert landscape, a change detection algorithm is used to identify the changes in green spaces. This algorithm includes the presentation of multitemporal datasets to evaluate the chronological special effects. This paper presents an optical flow analysis among images captured at different time sequences. The algorithm shows promising results of change detection in green patches in the desert of Saudi Arabia detected by color segmentation. The algorithm has been validated over a set of satellite images demonstrating an effective performance.
\end{abstract}

Keywords-Image processing; change detection; optical flow analysis; green patches; color segmentation

\section{INTRODUCTION}

In recent years, The Kingdom of Saudi Arabia has taken considerable strides to achieve food security, a goal that is one of the noble goals of Vision 2030. One of the strategies in achieving food security is to increase the agricultural area within the 2.15 million $\mathrm{km}^{2}$ (approx.) of the total area of the Kingdom of Saudi Arabia [1]. Although desert covers most of the Saudi Arabian land, historical images of Google Earth show a spread of green patches over the years. This observation was also reported by NASA in 2012 [2]. Some of those green patches are natural and others are artificial. The rationale behind this research is to contribute towards achieving food security in Saudi Arabia through detecting the spread of green patches using Change Detection (CD) algorithm. CD is the method of discovering variations or changes that occur in a series of images over a period of time. $\mathrm{CD}$ includes the presentation of datasets based on time series, thus, evaluating the evaluating the unique chronological properties. For the previous decades, Remote Sensing Data, for example, Thematic Mapper (TM) [3-5] and Satellite Probatoire d'Observation de la Terre (SPOT) [6], were the foremost sources of data for a number of CD applications. This is due to the benefits of: (1) data attainment that is performed repeatedly, (2) the synoptical assessment, and (3) the ability for computers to process digital data.

In this research, the proposed algorithm is implemented for sequences of satellite images for optical flow analysis. The images' capture, ranges from 1990 to 2020 depending on the region and on the available satellite records. The results exhibit a promising outcome of green patches detected using color segmentation.

The rest of this paper is organized as the following: Section 2 discussed some related work, particularly, in landscape analysis of green patches and the implementation of $\mathrm{CD}$ algorithms. The detailed implementation of the algorithm used in this paper is described in Section 3. The results are presented in Section 4, and finally this paper is concluded in Section 5.

\section{RELATED WORK}

\section{A. Landscape Analysis of Green Patches}

The author in [7] used the city of Hangzhou in China to validate their methodology, which maps landscape evaluation with the sub-pixel in order to investigating the spatiotemporal sequence that might emerge. Their methodology also examines the changes of classified green patches of urban areas. To deriving the area of green patches at the level of sub-pixel, the method examines (more than once) the spectral mixture that was used within the dataset of time series Landsat. Analyzing the landscape measure was performed to distinguishing the various green patches sequences of urban areas. Based on their methodology, a green space network was shaped, aiming to increase the green patches in the urban areas of Hangzhou. According to the authors, their study results should assist in refining the perceptive of the growth of assorted urban areas.

The author in [8] proposed an automated algorithm called Density Weighted Connectivity of Grass Pixels (DWCGP) in order to evaluate the roadside grass biomass from images to identify fire-prone regions. This method computes the vertical pixels length of connected grass in each column of a given image. After this step, the algorithm weights the length using the density of the grass in the column's region. Pixels classification is then conducted by utilizing Feedforward Artificial Neural Networks, whereas the main feature alignment at each pixel is calculated using multi-orientation Gabor Wavelet Filter vote. Their algorithm works well with

This research was sponsored by the Deputyship for Research \& Innovation, Ministry of Education in Saudi Arabia. 
grass stems that are not vertical, also it adapts to variations of Gabor filter parameters and surrounding region widths.

A fuzzy logic approach was conducted using Hadoop Hive [9]. This approach discovers the harvests plantation information from the agro-climatic historical database during the period of 1983-2016 taking Egypt as the test ground. Their approach gives a number of circumstances for the plantation dates for each harvest with a suitability level for every situation. Their proposed approach should help managing the procedure of harvests plantation, taking into consideration factors like: harvesting dates, diseases and watering requirements data.

In a contrary work [10], the authors conducted a study that evaluates the variations of drought in the Tihama Plain in Yemen that contributes towards over $40 \%$ of agricultural products in the country. This study considers the time span and its in relation to the drought spread. In their work, the Standardized Precipitation Index (SPI) was implemented in order to assess the status of drought temporally. Geographic Information Systems (GIS) was also implemented to display the different spreading of drought spatially in the region of interest (study area). Their results show a concerning drought phenomenon over three decades of time.

The author in [11] investigated the green field detection methods for visible satellite images using the vegetative index (COM) in addition to the development of two methods to increase detection accuracies, which are enhanced versions of COM. The visible satellite images included six locations and four zoom levels obtained through Google Map. The three methods were applied in their experiment in order to compare the accuracies in detection.

\section{B. Implementation of Change Detection Algorithms}

The author in [12] proposed an algorithm to generate the Landsat Burned Area Essential Climate Variable (BAECV) by recognizing burned spaces in dense time-series of Landsat data. Gradient boosted regression models were utilized in the generation of possible burn spaces by implementing band values and spectral indices from separate Landsat views, insulated surroundings, and varied measurements between the view and insulated surroundings. By utilizing pixel-level thresholding in conjunction with an area increasing procedure, the categorizations of burn were produced from the burn possibility surfaces. 1984 to 2015 was the time duration in which the BAECV results were produced for, using the United States (US) as the test ground. For each Landsat scene, the BAECV products were comprised of pixel-level burn probabilities, as well as annual combination that include the maximum burn probability and a burn organization.

The author in [13] proposed a collective of time series algorithms aiming to improve the observation of land change. Their methodology combines the algorithms of Continuous CD and Classification (CCDC) [14] and Cumulative Sum of Residuals (CUSUM) for break detection; for removing false positives (or breaks in time spans not demonstrating land change), the Chow Test [15] was utilized. The combination of those three algorithms, were implemented to three Landsat views taking the US as the test ground. The results were measured according to their capability in properly distinguish structural breaks from steady time spans.

The work presented in [16] display some initial results for CD by utilizing Landsat and Worldview images. The area of interest that the research targeted had some substantial development between May 2014 and October 2015 like buildings emerging. The authors investigated several methodologies for CD. The authors implemented pansharpening to improve the resolution to fifteen meters, and that was related to Landsat images. A chronochrome covariance equalization was also implemented between two Landsat images. The authors then analysed the residual between the two equalized images by utilizing various algorithms, for example, direct subtraction and global ReedXiaoli (GRX) detector.

The author in [17] applied images for Synthetic Aperture Radar (SAR) in order to observe disasters and for the purpose of environmental monitoring. The aim of their work is to minimize the effect of noise on SAR image CD. They built their work on mathematical morphology filtering and K-means clustering for SAR image CD. As an initial step, the authors used logarithmic transformation to transform multiplicative noise into additive noise in two SAR images. Then morphological filtering was used to de-noise the two multitemporal SAR images. Finally, in order to find the two distinctive images, the authors used the mean ratio operator and subtraction operator.

The author in [18] have proposed a Time-Series Classification approach based on CD (TSCCD) for rapid Land Use/Land Cover (LULC) mapping, which utilizes the Prophet algorithm in order to find the ground cover change points, then implement time sequence dissection in a time component and the Dynamic Time Warping (DTW) algorithm in order to categorize the sub-time sequence. Time sequence images of the City of Wuhan were selected for testing during the period of 2000 to 2018. For validation, the authors utilized China's national land-use surveys for the years: 2000, 2005, 2008, 2010, 2013, and 2015. In their research, they assumed that the ground cover stays unaffected in each subsequence, only one time-training sample selection and one LULC categorization were required in order to improve the effectiveness of their work. According to the authors, the Prophet can spot large and slight variations to a precise degree, capture the direction and rate of change, as well as its suitability to process noise and missing data. Because data is subject to irregular observations or missing values, DTW is primarily utilized to enhance the accuracy of time sequence categorization and to fix the time misalignment issues of ground cover series data.

Another algorithm was proposed in the CD field taking the urban renewal in the City of Guangzhou (China) as a test ground for the period between 2000 and 2010 [19]. Their research suggests a technique to extract and map yearly Impervious Surface Percentage (ISP) and to differentiate patterns in urban growth by utilizing time sequence images of average resolution. Their research was carried on by applying the Cubist tree model for annual ISP inversion (AoCubist), enhancing multi-temporal Landsat merged images to reduce the influence of phenology and inter-year climate change. They 
also developed the C5.0 decision tree algorithm with temporalspatial filtering guidelines in order to enhance the space-time endurance and the separation of patterns derived by unsupervised K-means categorization. Their results were validated against Google Earth images, which indicate that their categorization achieved an overall accuracy of $88.32 \%$ to $90.85 \%$. Whereas, the yearly urban growth rate stayed between $4 \%$ and $10 \%$, while the yearly deurbanization rate ranged between $1 \%$ and $5 \%$.

The proposed methodology described in the next section differs from those discussed above. It is a combination of algorithms that are applied to detect green patches in the desert of Saudi Arabia using satellite images.

\section{MethodOLOGY}

Three CD algorithms are widely used to detect the changes in green spaces: (1) image differencing, (2) image ratioing, and (3) Principal Component Analysis (PCA) [20]. Image differencing is accomplished by taking the absolute value of the subtraction of the second image from the first image. Image ratioing is accomplished by calculating the ratio of the amount of green space covered in two registered images captured at different times.

However, this apparent intensity change at each pixel is due to various factors like camera movement, change in position and intensity of light sources, object movement, deformation, specular reflections, and so on.

Although the image differencing is a simple method of CD, but it produces only the magnitudes of the changes. Ratioing is unable to describe changes efficiently in the region of interest (the study area). It produces partial change information and provides the least accuracy in comparison to other methods, thus in plane areas, it is discouraged. PCA approaches are employed for CD in sort of vegetation and water feature.

This research presents an optical flow analysis among images captured at different time sequences. Optical flow is given as the movement of discrete pixels within the area of an image. Optical flow usually gives a reasonable estimation of the actual physical motion applied within the area of an image [21].

The main idea of optical flow is derived from Lucas and Kanade [22], who presented an iterative registration technique for stereo employing motion detection called the local method of optical flow. Horn and Schunk [23] measured the optical flow using a global method which offers the best density in the optical flow. The popular optical flow spatiotemporal method of Black and Anandan [24] was one of the methods that were based on [22] and [23]. The author in [25] presented a combined global and local optic flow techniques which conglomerates the benefits of global and local methods. This research presents a Local Global Hybrid (LGH) method which employs the Lucas and Kanade's least-square fit approach a prototype of the local method, whereas the Horn and Schunck approach was utilized as a characteristic for a global method.

\section{A. Optical Flow Analysis}

Let $\left\{f_{1}, f_{2}, \ldots, f_{N}\right\}$ be a set of $N$ image sequences received at time $\left\{t_{1}, t_{2}, \ldots, t_{N}\right\}$ over satellite imagery. Every image maps a two-dimensional pixel coordinate $(x, y) \in R^{k}$ representing the brightness or color ( $k=1$ for gray-scale images and $k=3$ for RGB color images). A motion vector is determined by considering a correspondence between rectangles at times $\left\{t_{1}, t_{2}, \ldots, t_{N}\right\}$, where $t$ is the time index in image sequences:

$m_{v}=\arg \min \| f_{1}(x, y, t)-f_{2}(x-a, y-b, t+1 \|$

Most of the researcher use block-matching algorithm to determine the motion vector. Nevertheless, the main drawback of using block-matching algorithm for determining the motion vector is that its computational cost is high. $N^{2}$ different possible velocity vectors have to be checked in the image, each of which requires $N^{2}$ additions for every block. Numerous suboptimal search strategies try to obtain the approximate velocity vector, without really evaluating all $N^{2}$ of the possible velocity vectors. Optical flow deliberates the motion vector as a function of continuous position, rather than discrete position.

The Horn-Schunck technique of approximating optical flow is a global approach which presents a global restriction of smoothness. The flow is expressed by a global energy function that is required to be reduced:

$$
E=\iint\left[\left(f_{x} a+f_{y} b+f_{t}\right)^{2}+\alpha^{2}\left(|\nabla a|^{2}+|\nabla b|^{2}\right)\right] d x d y
$$

where $f_{x}, f_{y}$ and $f_{t}$ are the derivatives of the image intensity values along the $x, y$ and time dimensions respectively. The optical flow vector is $\mathbf{v}=[a, b]^{T}$, and the parameter $\alpha(>0)$ is a regularization constant.

This research outlines a Local-Global Hybrid (LGH) approach for optical flow analysis with the following notations:

$$
\begin{aligned}
& w=(a, b, t)^{T} \\
& |\nabla w|^{2}=|\nabla a|^{2}+|\nabla b|^{2} \\
& \nabla_{3} f=\left(f_{x}, f_{y}, f_{z}\right)^{T} \\
& J \rho\left(\nabla_{3} f\right)=k \rho^{*}\left(\nabla_{3} f \nabla_{3} f^{T}\right)
\end{aligned}
$$

Obviously, the Lucas-Kanade approach reduces the quadratic form given by the following equation:

$$
J \rho\left(\nabla_{3} f\right)=k \rho *\left(\nabla_{3} f \nabla_{3} f^{T}\right)
$$

The Horn-Schunck approach reduces the function given below:

$$
E_{H S}(w)=\int_{\Omega}\left[w^{T} J_{0}\left(\nabla_{3} f\right) w+\alpha\left(|\nabla w|^{2}\right)\right\rfloor d x d y
$$


This expression recommends a likely technique to spread out the Horn-Schunck equation to the required LGH method. Once the matrix $J_{0}\left(\nabla_{3} f\right)$ is replaced by the structure tensor $J_{\rho}\left(\nabla_{3} f\right)$ with selected integration scale $\rho>0$, (9) is established.

$E_{L G H}(w)=\int_{\Omega}\left[w^{T} J_{\rho}\left(\nabla_{3} f\right) w+\alpha\left(|\nabla w|^{2}\right)\right] d x d y$

The reduced flow field $(a, b)$ fulfills the Euler- Lagrange equations expressed by the following equations:

$$
\begin{aligned}
& 0=\Delta a-\frac{1}{\alpha}\left(K_{\rho} * f_{x}^{2} a+K_{\rho} * f_{x} f_{y} b+K_{\rho} * f_{x} f_{t}\right) \\
& 0=\Delta b-\frac{1}{\alpha}\left(K_{\rho} * f_{y}^{2} b+K_{\rho} * f_{x} f_{y} a+K_{\rho} * f_{x} f_{t}\right)
\end{aligned}
$$

The aforementioned methods applied were merely the spatial smoothness functions. However, the spatiotemporal expressions provide more suitable results than spatial expressions due to the extra de-noising belongings in the temporal direction [26]. A spatiotemporal alternate of the Lucas-Kanade method just substitutes the convolution of twodimensional Gaussians by spatiotemporal convolution of threedimensional Gaussians. This leads to a 2-by-2 linear system of equations for $\mathrm{a}$ and $\mathrm{b}$ that are unknown. Combining the LucasKanade's temporal extended variant method with the HornSchunck method, spatiotemporal version of the proposed LGH function is established (12) [26].

$$
E_{L G H 3}(w)=\int_{\Omega \times[0, T]}\left\lfloor w^{T} J_{\rho}\left(\nabla_{3} f\right) w+\alpha\left(|\nabla w|^{2}\right)\right\rfloor d x d y
$$

where convolutions with Gaussians are performed in a spatiotemporal approach and it is given below:

$\left|\nabla_{3} w\right|^{2}=\left|\nabla_{3} a\right|^{2}+\left|\nabla_{3} b\right|^{2}$

If $J_{p q}$ is the component $(p, q)$ of the structure tensor, $J_{\rho}\left(\nabla_{3} f\right)$, the Euler-Lagrange equations are provided by the equations below:

$$
\begin{aligned}
& 0=\nabla_{3} a-\frac{1}{\alpha}\left(J_{11} a+J_{12} b+J_{13}\right) \\
& 0=\nabla_{3} b-\frac{1}{\alpha}\left(J_{21} a+J_{22} b+J_{23}\right)
\end{aligned}
$$

If the spatiotemporal Laplacian substitutes the spatial Laplacean, then the following equation will be achieved.

$\Delta_{3}=\partial_{x x}+\partial_{y y}+\partial_{11}$

\section{B. Proposed Algorithm for $L G H$}

For a rectangular pixel grid, functions $a(x, y, t)$ and $b(x, y, t)$ are unknown, whereas the $a$ estimation at some pixel $k$ with $(k=1, \ldots, N)$ can be considered as $a_{k}$. If $J_{p q k}$ is the component $(p, q)$ of the tensor $J_{\rho}(\nabla f)$ of pixel $k$ and $N(k)$ represents the set of ( 4 in 2D, 6 in 3D) neighbours of pixel $k$, so a finite difference estimation to the Euler-Lagrange equations are provided by the following:

$$
\begin{aligned}
& 0=\sum_{j \in N(k)} \frac{a_{j}-a_{k}}{h^{2}}-\frac{1}{\alpha}\left(J_{11 k} a_{k}+J_{12 k} b_{k}+J_{13 k}\right) \\
& 0=\sum j \in N(k) \frac{b_{j}-b_{k}}{h^{2}}-\frac{1}{\alpha}\left(J_{21 k} a_{k}+J_{22 k} b_{k}+J_{23 k}\right)
\end{aligned}
$$

where $k=1, . . ., N$. This sparse linear system of expressions has solved the Successive Over-Relaxation (SOR) methodology due to its simplicity and effectiveness [26]. When the upper index represents the iteration step, (18) and (19) can express the SOR method.

$$
\begin{aligned}
a_{k}^{i+1} & =(1-\omega) a_{k}^{i}+\omega \frac{\left.\sum_{j \in N^{-}(k)} a_{j}^{i+2}+\sum_{j \in N^{+}(k)} a_{j}^{i}\right)}{|N(k)|+\frac{h^{2}}{\alpha} J_{11 k}} \\
& -\omega \frac{\frac{h^{2}}{\alpha}\left(J_{12 k} b_{k}^{i}+J_{13 k}\right.}{|N(k)|+\frac{h^{2}}{\alpha} J_{11 k}}
\end{aligned}
$$

$$
\begin{gathered}
b_{k}^{i+1}=(1-\omega) b_{k}^{i}+\omega \frac{\left.\sum_{j \in N^{-}(k)} b_{j}^{i+2}+\sum_{j \in N^{+}(k)} b_{j}^{i}\right)}{|N(k)|+\frac{h^{2}}{\alpha} J_{22 k}} \\
-\omega \frac{-\frac{h^{2}}{\alpha}\left(J_{22 k} a_{k}^{i}+J_{23 k}\right.}{|N(k)|+\frac{h^{2}}{\alpha} J_{22 k}}
\end{gathered}
$$

where

$$
N^{-}(k)=\{k \in N(k), j<k\}, N^{+}(k)=\{k \in N(k), j>k\} .
$$

Here $|N(k)|$ represents the number of pixels that are adjacent to pixel $k$, which belong to the image area. The relaxation parameter $\omega \in[0,2]$ has a solid impact on the convergence speed. For $\omega=1$ it matches exactly with the Gauss-Seidel method. Normally, the typical values for $\omega$ are $[1.9,1.99]$. Considering the first iteration, the flow components are initiated by 0 . The explicit option of the initialization is not vital, this is because the method is globally convergent. The algorithm is applied for a number of image sequences with time frames $\left\{t_{1}, t_{2}, \ldots, t_{N}\right\}$. 


\section{Optical Flow Field Construction Algorithm}

Input: A set of images at times $\left\{t_{1}, t_{2}, \ldots, t_{N}\right\}$, iteration $i$ for SOR, $\alpha, w$ and $\rho$.

\section{Output: Optical Flow Field}

Method: The Optical Flow Field is created by these steps.

1. Read files in matrix array.

2. For each image received at time $t$, perform the following steps:

i. Pre-smooth the image by implementing the Gaussian Kernel with standard deviation $\alpha$.

ii. Compute the variation of image $f_{x}, f_{y}, f_{t}$.

iii. Calculate the velocity $a, b$ of image $t$ by using the (SOR) methodology for $k$ iteration.

iv. Compute the resulting velocity and angle, then draw.

v. Store the outcome.

3. End

\section{EXPERIMENTAL RESULTS}

The effectiveness of the algorithm has been validated over a set of satellite images. The images were collected from Google Earth. A sample image of the study area is shown in Fig. 1.

Experiments have been conducted using Visual C++ software in the Windows 10 operating system. The computer's setup was Intel ${ }^{\circledR}$ Core $^{\mathrm{TM}}$ i5 $\mathrm{CPU}$ with $2.70 \mathrm{GHz}$, and $2 \mathrm{~GB}$ RAM.

The classified map of the study area has been constructed, illustrating the surface cover using ArcGIS. Fig. 2 illustrates the dominant surface cover of the study area. The green spaces are presented in green colors, orange presents grounds, sand dunes are presented in yellow, buildings are presented in red, while the water bodies are presented in blue.

The outcome of the optical flow field generated by utilizing LGH methodology is presented in Fig. 3. This represents the set of (4 in 2D, 6 in 3D) neighbours of pixel that are adjacent to pixel $k$, which belong to the image area. This was applied to a number of image sequences with time frames.

Obviously, too much regularization reduces the flow of the changes. Optical flow outcomes better results when the regularization parameter $\alpha$ is from 0.05 to 0.09 for 200 to 1000 iterations. The green areas are finally detected by color segmentation in CIE $a^{*} b^{*}$ color model, as shown in Fig. 4.

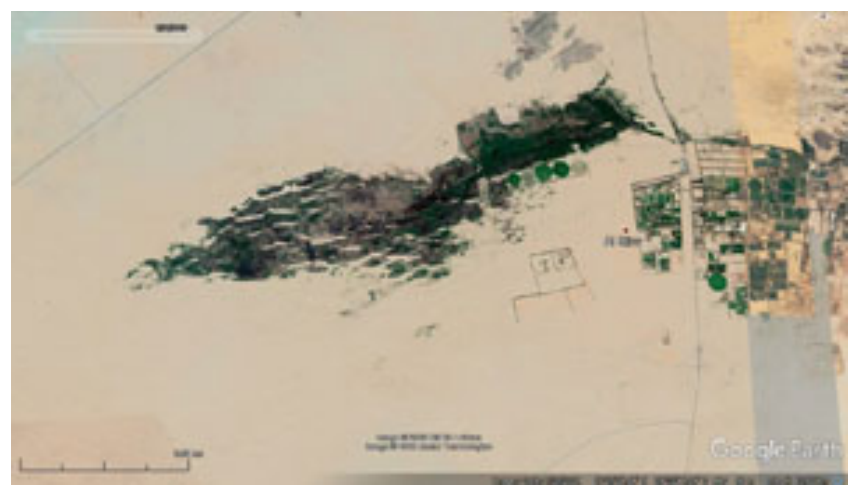

Fig. 1. A Sample Image of the Study Area.

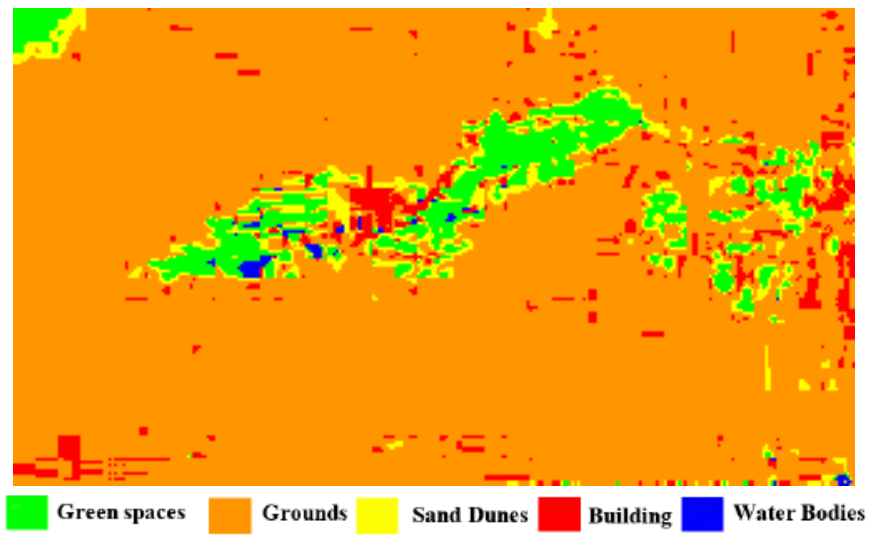

Fig. 2. A Sample Image Illustrating the Surface Cover.
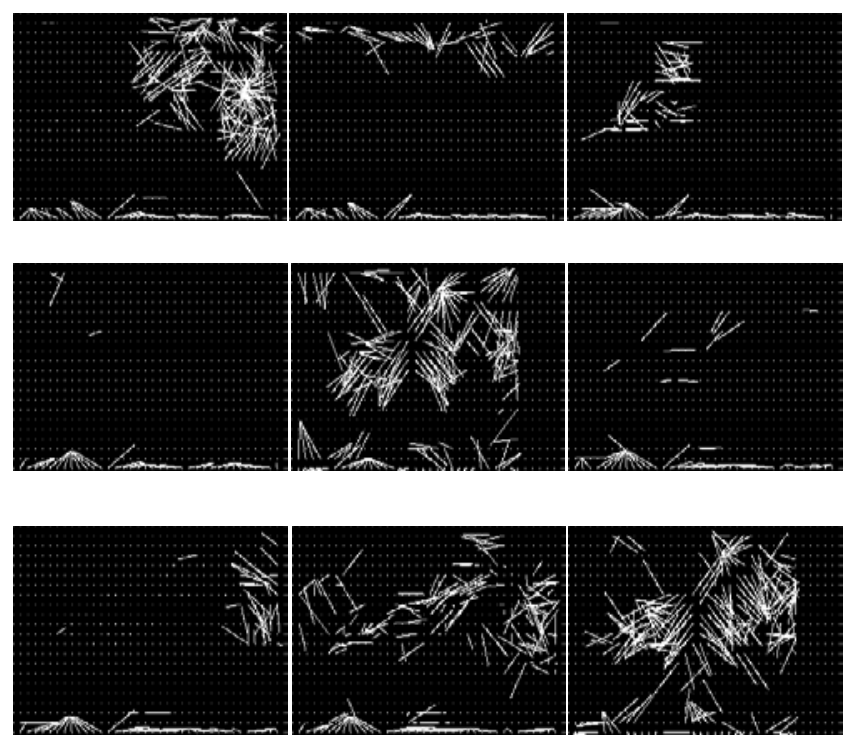

Fig. 3. Optical Flow Field Generated by utilizing LGH Methodology. 


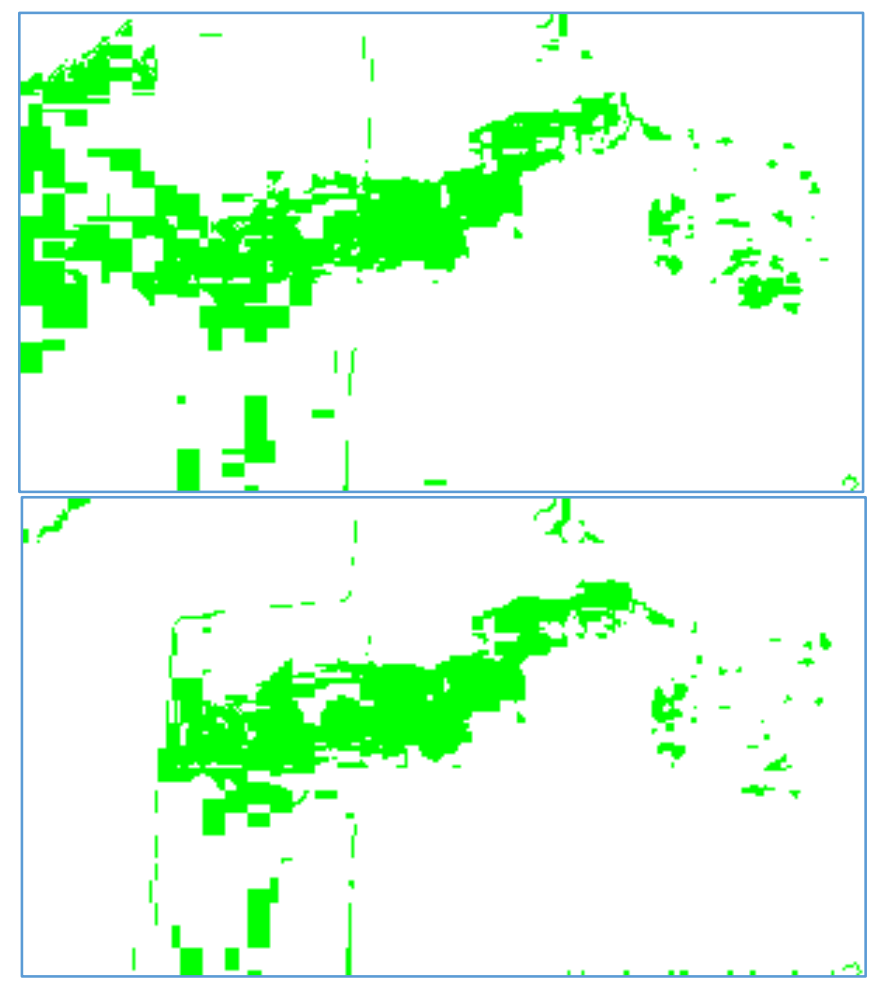

Fig. 4. Extraction of the Green Spaces from Surface Cover Images.

\section{CONCLUSION}

In order to detect the green patches in the desert of Saudi Arabia, a series of satellite images were captured that cover 2 to 3 decades of time depending on the location and on the available satellite images. CD algorithm was utilized to identify the changes in green spaces.

Optical flow analysis was implemented to the image sequences received at a certain time through the satellite imagery. Each image maps a 2D pixel coordinate representing the gray-scale or the RGB color images. A motion vector is determined by considering a correspondence between rectangles at give times.

This research outlines a Local-Global Hybrid (LGH) approach for optical flow analysis in a way that combines the temporal extended variant of both the Lucas-Kanade and the Horn-Schunck methods. The optical flow field was constructed using a set of images at given times and processed in a number of steps. The experimental results show an effective detection of the green patches from the desert area. This research will be conducted further in order to compute the rate of growth in the green patches of the Saudi desert, thus, observing a pattern that may emerge. This will then lead to further analyses and prediction of future spread of the green patches.

\section{ACKNOWLEDGMENT}

The authors extend their appreciation to the Deputyship for Research \& Innovation, Ministry of Education in Saudi Arabia for funding this research work through the project number IFT20164.

\section{REFERENCES}

[1] S. Fiaz, M. A. Noor, and F. O. Aldosri, "Achieving food security in the Kingdom of Saudi Arabia through innovation: Potential role of agricultural extension”. Journal of the Saudi Society of Agricultural Sciences, vol. 17, pp. 365-375, October 2018.

[2] “NASA Sees Fields of Green Spring up in Saudi Arabia”, date of last visit 09/11/2020: https:/www.nasa.gov/topics/earth/features/saudigreen.html.

[3] Y. Chen, Y. Wei, Q. Wang, F. Chen, C. Lu, and S. Lei, "Mapping PostEarthquake Landslide Susceptibility: A U-Net Like Approach”, Remote Sensing, vol. 12, pp. 2767(1-25), 2020.

[4] S. Obata, P. Bettinger, C.J. Cieszewski and R.C. Lowe, "Mapping Forest Disturbances between 1987-2016 Using All Available Time Series Landsat TM/ETM+ Imagery: Developing a Reliable Methodology for Georgia, United States”, Forests, vol. 11, pp. 335(1-16), 2020.

[5] A. Bannari and Z.M. Al-Ali, “Assessing Climate Change Impact on Soil Salinity Dynamics between 1987-2017 in Arid Landscape Using Landsat TM, ETM+ and OLI Data”, Remote Sensing, vol. 12, pp. 2794(1-18), 2020.

[6] X. Cheng, M.M. Nizamani, C.Y. Jim, K. Balfour, L. Da, S. Qureshi, Z. Zhu and H. Wang, "Using SPOT Data and FRAGSTAS to Analyze the Relationship between Plant Diversity and Green of Zhanjiang, China”, Remote Sensing, vol. 12, no. 3, pp. 3477( 1-16), 2020.

[7] Z. Yu et al., "Dynamics of Hierarchical Urban Green Space Patches and Implications for Management Policy”. Sensors, Basel, vol. 17, June 2017.

[8] L. Zhang, B. Vermaa, D. Stockwell, and S. Chowdhurya, "Density Weighted Connectivity of Grass Pixels in Image Frames for Biomass Estimation”. Expert Systems with Applications, vol. 101, February 2018.

[9] A. H. Mohammed, A. M. Gadallah, H. A. Hefny, and M. Hazman, "Fuzzy based approach for discovering crops plantation knowledge from huge agro-climatic data respecting climate changes”, Computing, vol. 100, pp. 689-713, April, 2018.

[10] A. A. Dhaif Allah, N. Bin MD. Hashim, and A. Bin Awang, "Discovering Trends of Agricultural Drought in Tihama Plain, Yemen: A Preliminary Assessment”, Indonesian Journal of Geography, vol. 49, pp. 17- 27, June 2017.

[11] A. Choukuljaratsiri, P. Chaovalit, and S. Pongnumkul, "Plant cover detection from visible satellite imagery”. IEEE International Computer Science and Engineering Conference (ICSEC). Thailand, pp. 1-6, November, 2015.

[12] T. J. Hawbaker et al., "Mapping burned areas using dense time-series of Landsat data”, Remote Sensing of Environment, vol. 198, pp 504-522, September 2017.

[13] E. L. Bullock, C. E. Woodcock, and C. E. Holden, "Improved change monitoring using an ensemble of time series algorithms", Remote Sensing of Environment, vol. 238, March 2020.

[14] Z. Zhu, and C. Woodcock, "Continuous Change Detection and Classification of Land Cover Using All Available Landsat Data”, Remote Sensing of Environment, vol. 144, pp. 152-171, March 2014.

[15] G. C. Chow, "Tests of Equality Between Sets of Coefficients in Two Linear Regressions”. Econometrica, vol. 28, pp. 591-605, July 1960.

[16] C. Kwan et al., "Change detection using Landsat and Worldview images", Proc. SPIE. USA, vol. 10986, May 2019.

[17] L. Liu, Z. Jia, J. Yang, and N. K. Kasabov, "SAR Image Change Detection Based on Mathematical Morphology and the K-Means Clustering Algorithm," in IEEE Access, vol. 7, pp. 43970-43978, 2019.

[18] J. Yan et al., "A time-series classification approach based on change detection for rapid land cover mapping," ISPRS Journal of Photogrammetry and Remote Sensing, vol. 158, pp. 249-262, December 2019.

[19] Y. Fu et al., "Characterizing the spatial pattern of annual urban growth by using time series Landsat imagery," Science of The Total Environment, vol. 666, pp. 274-284, May 2019.

[20] S. Minu and A. Shetty, “A comparative study of image change detection algorithms in MATLAB”, Aquatic Procedia, vol. 4, pp. 1366-1373, 2015. 
[21] P. Turaga, R. Chellappa, and A. Veeraraghavan, "Advances in VideoBased Human Activity Analysis: Challenges and Approaches," Advances in Computers, vol. 80, pp. 237-290, 2010.

[22] B.D. Lucas and T. Kanade, “An Iterative Image Registration Technique with an Application to Stereo Vision", Proceedings of the DARPA Image Understanding Workshop, Washington, DC, USA, pp. 674-679, April 1981.

[23] B. Horn and B. Schunck, "Determining optical flow", Artificial Intelligence, vol. 7, pp.185-203, 1981.
[24] M.J. Black and P. Anandan, "The robust estimation of multiple motions: Parametric and piecewise-smooth flow fields", Computer Vision and Image Understanding, vol. 63, pp. 75-104, 1996.

[25] B. Andres, F.A. Hamprecht, and C.S. Garbe, "Selection of Local Optical Flow Models byMeans of Residual Analysis”, AGM 2007, LNCS 4713, pp. 72-81, 2007.

[26] K.Md. Shahiduzzaman, K.M. Reza, and N. Tazin, "Combined Local Global Optical Flow Approach in Bio-medical Image Sequences for blood flow detection”, International Journal of Advanced Research in Computer and Communication Engineering, vol. 4, pp.468-472, February 2015. 